\title{
COMPACTNESS AND CONNECTEDNESS AS COMPOSABLE PROPERTIES
}

\author{
T. B. MUENZENBERGER AND R. E. SMITHSON
}

(Received 22 February 1972)

Communicated by G. B. Preston

\section{Introduction}

In [5] Magill showed that compactness is a composable property of Hausdorff spaces. (That is, if $\alpha, \beta$ are compact subsets of $X \times X$, then $\alpha \circ \beta$ is also compact when $X$ is $T_{2}$.) Also Magill gave an example to show that the composition of connected relations need not be connected. Subsequently, he characterized Hausdorff $k$-spaces in terms of the semigroup of compact relations in $X$.

The purpose of the present note is to obtain an analogous characterization of semi-locally connected, $R_{0}$-spaces in terms of the relations which preserve connectedness. We also give another proof of the above stated result of Magill, and then obtain an equivalent formulation of his result that sheds considerable light on the connectedness situation. We then use the new technique to obtain an extension of some of Magill's results to non-Hausdorff spaces. Finally, we combine compactness and connectedness to obtain a new composable property and to characterize semi-locally connected, $c c$-spaces.

Let $X$ be a set and let $\alpha, \beta \subseteq X \times X$ be relations in $X$. Then

$$
\alpha \circ \beta=\{(x, y) \in X \times X \text { : there exists a } z \in X \text { such that }(x, z) \in \beta \text { and }(z, y) \in \alpha\} \text {. }
$$

A set of relations $S$ in $X$ is a semigroup of relations is case $\alpha, \beta \in S$ implies that $\alpha \circ \beta \in S$. If $\alpha \subseteq X \times X$, then the domain of $\alpha$ is denoted by $D(\alpha)$ and the range by $R(\alpha)$.

In [4] a triform was defined as a triple $\left(X, \mathscr{F}_{X}, S[X]\right)$ where $X$ is a nonempty set, $\mathscr{F}_{X}$ is a family of subsets of $X, S[X]$ is a set of relations in $X$, and the following conditions are satisfied:

(i) For each $x \in X,\{(x, x)\} \in S[X]$.

(ii) $H \in \mathscr{F}_{X}$ if and only if $H \times\{x\} \in S[X]$ for some $x \in X$.

(iii) If $\alpha, \beta \in S[X]$, then $\alpha \circ \beta \in S[X]$. 
From (iii) we note that $S[X]$ is a semigroup of relations, and $S[X]$ is called a triform semigroup. We shall use the following theorem concerning bijections on triform semigroups. The statement of Theorem $A$ is as in [5], but the proof is in [4].

THEOREM A. Let $S[X]$ and $S[Y]$ be two triform semigroups and let $\theta$ be a bijection on $S[X]$ onto $S[Y]$. Then the following are equivalent:

(a) $\theta$ is an isomorphism.

(b) There exists a bijection $h$ from $X$ onto $Y$ such that $h(A) \in \mathscr{F}_{Y}$ for each $A \in \mathscr{F}_{X}, h^{-1}(A) \in \mathscr{F}_{X}$ for each $A \in \mathscr{F}_{Y}$, and $\theta(\alpha)=h \circ \alpha \circ h^{-1}$ for each $\alpha \in S[X]$.

(c) There exists a bijection $h$ from $X$ onto $Y$ such that $h(A) \in \mathscr{F}_{Y}$ for each $A \in \mathscr{F}_{X}, h^{-1}(A) \in \mathscr{F}_{X}$ for each $A \in \mathscr{F}_{Y}$, and $\theta(\alpha)=\{(h(x), h(y)):(x, y) \in \alpha\}$ for each $\alpha \in S[X]$.

\section{Connectedness}

In this section we characterize certain spaces in terms of the relations in the space which preserve connected sets both ways and have connected domain. We shall see that this is appropriate in section 3. If $\alpha \subseteq X \times X$, and if $A \subseteq X$, then

$$
\alpha(A)=\{y:(x, y) \in \alpha \text { for some } x \in A\} .
$$

Note that $\alpha \circ \beta(A)=\alpha(\beta(A))$ when $\beta$ is another relation in $X$.

Now let $S$ be the relations $\alpha$ in $X$ such that $D(\alpha)$ is connected and such that $\alpha(A)$ and $\alpha^{-1}(A)$ are connected whenever $A$ is connected. Further, let $\mathscr{F}$ be the set of connected subsets of $X$. (Note that $\phi \in \mathscr{F}$ and $\alpha(\phi)=\phi$ for all $\alpha \subseteq X \times X$ where $\phi$ is the empty set.) Then with these sets we obtain the following theorem.

THEOREM 2.1. The triple $(X, \mathscr{F}, S)$ is triform.

Proof. Clearly $\{(x, x)\} \in S$ for all $x \in X$. If $\alpha=H \times\{x\}$ where $H \in \mathscr{F}$ and if $A \subseteq X$, then $D(\alpha)=H, \alpha(A) \subseteq\{x\}, \alpha^{-1}(A)=H$ if $x \in A$, and $\alpha^{-1}(A)=\phi$ if $x \notin A$. Thus $\alpha \in S$. Conversely, if $\alpha=H \times\{x\} \in S$, then $\alpha^{-1}(x)=H$ and so $H \in \mathscr{F}$. Thus $H \times\{x\} \in S$ if and only if $H \in \mathscr{F}$. Let now $\alpha, \beta \in S$. Then $D(\alpha \circ \beta)$ $=\beta^{-1}(D(\alpha)) \in \mathscr{F}$. If $A$ is a connected subset of $X$, then $\alpha \circ \beta(A)=\alpha(\beta(A))$, and since $\beta(A)$ is connected, $\alpha(\beta(A))$ is connected. Similarly, $(\alpha \circ \beta)^{-1}(A)$ $=\beta^{-1}\left(\alpha^{-1}(A)\right)$ is also connected. Thus $S$ is a semigroup of relations.

Remarks. If $\alpha \in S$, then $R(\alpha)=\alpha(D(\alpha))$ is connected and so $\alpha \in S$ if and only if $\alpha^{-1} \in S$. We could replace the set $S$ defined above with either of the following two sets:

$$
\begin{aligned}
& S^{\prime}=\left\{\alpha \subseteq X \times X: \alpha(A) \text { and } \alpha^{-1}(A) \text { are connected for all connected } A \subseteq X\right\}, \\
& S^{\prime \prime}=\left\{\alpha \subseteq X \times X: \alpha^{-1}(A) \text { is connected for all connected } A \subseteq X\right\} .
\end{aligned}
$$


Then the triples $\left(X, \mathscr{F}, S^{\prime}\right)$ and $\left(X, \mathscr{F}, S^{\prime \prime}\right)$ are triform. Observe that $S \subseteq S^{\prime} \subseteq S^{\prime \prime}$. From the proof of Theorem 2.1, $S^{\prime \prime}$ is a maximal triform.

Before proceeding we give some definitions and examples.

Definitions. A multifunction is a relation on a set $X$ into a set $Y$. (A relation is a multifunction on its domain.) Let $F: X \rightarrow Y$ be a multifunction on the topological space $X$ into the topological space $Y$. Then $F$ is upper semicontinuous (u.s.c.) in case

$$
F^{-1}(B)=\{x \in X: F(x) \cap B \neq \phi\}
$$

is closed whenever $B$ is a closed subset of $Y$. Further, $F$ is point closed (compact, connected) if $F(x)$ is closed (compact, connected) for each $x \in X$. Finally, $F$ is continuum valued just in case $F(x)$ is a continuum for each $x \in X$.

EXAMPLE 1. Let $X$ be the reals with the usual topology and set

$$
\alpha=\{(x, 1 / x): x \in X \text { and } x \neq 0\} \cup\{(0, y): y \in X\} .
$$

Then $\alpha$ is point connected and u.s.c. and therefore $\alpha$ preserves connected sets. Also $D(\alpha)$ and $R(\alpha)$ are connected. But $\alpha^{-1}$ does not preserve connected sets and $\alpha$ is not connected. Observe that $\alpha(0)$ is not compact. This must occur since an u.s.c. continuum valued multifunction with connected domain is connected.

EXAMPLE 2. Let $X$ be the unit interval with the usual topology and set

$$
\alpha=\{(x, 0) \mid 0 \leqq x \leqq 1 / 2\} \cup\{(1 / 2, y) \mid 0 \leqq y<1\} \cup\{(x, 1) \mid 1 / 2<x \leqq 1\} .
$$

Then $\alpha, \alpha^{-1}$ preserve connected sets and $\alpha$ has connected domain. So $\alpha \in S$, but $\alpha$ is not connected. Conversely, $\alpha$ connected does not even imply that $\alpha$ is point connected (for example, a circle).

Example 3. Let $X$ and $\alpha$ be as in example 1 and let $\beta=\{(1,0)\}$. Then $\alpha, \beta$ preserve connected sets and have connected domain, but $D(\beta \circ \alpha)=\{0,1\}$ is disconnected. This shows the necessity of assuming that the inverse function preserves connected sets if one requires connected domains.

By example 2 the set of connected relations is not related to $S$ under inclusion. The importance of this remark will become evident in section 3 .

In order to give the characterization of certain topological spaces in terms of the semigroup of relations $S\left(S^{\prime}\right.$ or $\left.S^{\prime \prime}\right)$, we need a result of Sanderson [6]. For this we use the following definitions.

Definitions. A topological space $X$ is an $R_{0}$-space in case distinct point closures are disjoint. (Note that a $T_{1}$-space is $R_{0}$.) Further, a function $f: X \rightarrow Y$ is called biconnected in case $f$ is one-to-one and both $f$ and $f^{-1}$ preserve connected sets. 
LEMMA 2.2. A biconnected function onto a semi-locally connected, $R_{0}$-space is continuous.

Remarks. The definition of an $R_{0}$-space is due to Davis [1], and the definition of a biconnected function is due to Tanaka [7]. Observe that Lemma 2.2 includes the locally connected, locally compact $T_{2}$-spaces and hence the trees. As a consequence of Lemma 2.2, among such spaces homeomorphisms coincide with biconnected functions.

Lemma 2.2 is a corollary of the main results in [6], and its proof is omitted.

Let $S[X](S[Y])$ be the semigroup of relations in $X(Y)$ such that $\alpha \in S[X]$ $(S[Y])$ if and only if $\alpha \subseteq X \times X(Y \times Y), D(\alpha)$ is connected, and $\alpha, \alpha^{-1}$ preserve connected sets. One makes similar definitions of $S^{\prime}[X]$ and $S^{\prime \prime}[X]\left(S^{\prime}[Y]\right.$ and $\left.S^{\prime \prime}[Y]\right)$ that correspond to our earlier definitions of $S^{\prime}$ and $S^{\prime \prime}$.

THeOREM 2.3. Let $X, Y$ be semi-locally connected, $R_{0}$-spaces. If $S[X]$ and $S[Y]$ are isomorphic, then $X$ and $Y$ are homeomorphic, and conversely.

Proof. Let $S[X]$ and $S[Y]$ be isomorphic. By Theorem A there is a bijection $h$ on $X$ onto $Y$ such that $h(A)$ is connected for all connected subsets $A$ of $X$ and $h^{-1}(B)$ is connected for all connected subsets $B$ of $Y$. Thus, by Lemma $2.2, h$ and $h^{-1}$ are continuous. Hence, $X$ is homeomorphic to $Y$. Conversely, if $h: X \rightarrow Y$ is a homeomorphism, then $S[X]$ and $S[Y]$ are isomorphic by Theorem $A$ because $\alpha \in S[X]$ implies

$$
h \circ \alpha \circ h^{-1} \in S[Y] .
$$

For clearly $h \circ \alpha \circ h^{-1}$ and $h \circ \alpha^{-1} \circ h^{-1}$ preserve connected subsets of $Y$ if $\alpha, \alpha^{-1}$ preserve connected subsets of $X$. Also

$$
D\left(h \circ \alpha \circ h^{-1}\right)=h(D(\alpha))
$$

is connected if $D(\alpha)$ is connected. This completes the proof.

Also a semi-locally connected, $R_{0}$-space $X$ is characterized topologically by either of $S^{\prime}[X]$ or $S^{\prime \prime}[X]$. In fact any triform semigroup $S_{0}[X]$ with the following property will serve to characterize $X$ topologically:

If $h: X \rightarrow Y$ is a homeomorphism on $X$ onto another space $Y$, then $\alpha \in S_{0}[X]$ implies $h \circ \alpha \circ h^{-1} \in S_{0}[Y]$ (compare Proposition 3.6 of [5]).

Remark. The diagonal $\Delta=\{(x, x): x \in X\}$ is clearly an identity for $S^{\prime}[X]$ and $S^{\prime \prime}[X]$. Moreover, if $S[X]$ has an identity, it must be $\Delta$. Thus we get

Proposition 2.4. The semigroup of relations $S[X]$ has an identity if and only if $X$ is connected. 


\section{Compact relations}

In this section we give a new proof that compact relations in Hausdorff spaces are composable in the sense of Magill [5], and we extend Magill's results to non-Hausdorff spaces. For this we need some basic results from the theory of multifunctions. The first lemma follows directly from the definition and its proof is omitted.

LemMa 3.1. A multifunction $F: X \rightarrow Y$ is u.s.c. if and only if whenever $F(x) \subseteq U$, an open subset of $Y$, there exists an open set $V \subseteq X$ containing $x$ such that $F(V) \subseteq U$.

LEMMA 3.2. If $F: X \rightarrow Y$ is a point compact, u.s.c. multifunction and if $A \subseteq X$ is compact, then $F(A)$ is compact.

Proof. Let $\mathscr{U}$ be an open cover of $F(A)$. Then for each $x \in X$ there is a finite subset $U_{1}(x), \cdots, U_{k}(x)$ of $\mathscr{U}$ such that

$$
F(x) \subseteq \bigcup_{i=1}^{k} U_{i}(x) .
$$

Then since $F$ is u.s.c., we obtain an open set $V \subseteq X$ such that $x \in V$ and

$$
F(V) \subseteq \bigcup_{i=1}^{k} U_{i}(x) \text {. }
$$

Then, since $A$ is compact, there is a finite subcover $V_{1}, \cdots, V_{m}$ of $A$ of such open sets. Then the corresponding set

$$
\left\{U_{i}\left(x_{j}\right): i=1, \cdots, k_{j} ; j=1, \cdots, m\right\}
$$

is a finite subcover of $F(A)$.

LEMMA 3.3. If $F: X \rightarrow Y$ and $G: Y \rightarrow Z$ are u.s.c. multifunctions, then $H=G \circ F: X \rightarrow Z$ is u.s.c. Moreover, if both $F, G$ are point compact, then so is $H$. 3.2 .

ProOf. For the first part apply the definition. For the second part use Lemma

LEMMA 3.4. If $F: X \rightarrow Y$ is an u.s.c., point compact multifunction with compact domain, then $F$ is a compact subset of $X \times Y$. Moreover, if $X$ and $Y$ are $T_{2}$, then $F$ is a closed subset of $X \times Y$.

Proof. Let $\mathscr{U}$ be an open cover of $F$ in $X \times Y$. Since $F$ is point compact, $\{x\} \times F(x)$ is a compact subset of $F$. Thus there is a finite subcover $\left\{U_{i}(x): i\right.$ $\left.=1, \cdots, k_{x}\right\}$ of $\{x\} \times F(x)$. Hence, by a theorem of A. D. Wallace (see Kelley [2], page 142, Theorem 12), there exist open sets $V \subseteq X$ and $W \subseteq Y$ such that

$$
x \in V, F(x) \subseteq W \text { and } V \times W \subseteq \cup\left\{U_{i}(x): i=1, \cdots, k_{x}\right\} .
$$


Since the domain of $F$ is compact, we obtain a finite cover $V_{1}, \cdots, V_{n}$ (with corresponding sets $W_{1}, \cdots, W_{n}$ and finite collections $\left.\left\{U_{i, j}: i=1, \cdots, k_{j}\right\}\right)$ of $D(F)$. Then the collection

$$
\left\{U_{i, j}: j=1, \cdots, n ; i=1, \cdots, k_{j}\right\}
$$

is a finite subcover of $\mathscr{U}$ for $F$.

Note. Lemma 3.4 was stated so that it will apply even when the domain of $F$ is not all of $X$.

LEMma 3.5. Suppose the compact subsets of $X$ are closed. If $F \subseteq X \times Y$ is compact, then $F$ is u.s.c. and point compact.

Proof. Let $A \subseteq Y$ be closed and let $\pi_{1}, \pi_{2}$ be the projections on $X \times Y$ onto $X$ and $Y$, respectively. Then $\pi_{2}{ }^{-1}(A)$ is closed and so $\pi_{2}{ }^{-1}(A) \cap F$ is compact. Thus

$$
\pi_{1}\left(\pi_{2}{ }^{-1}(A) \cap F\right)=F^{-1}(A)
$$

is compact; hence, closed. Thus $F$ is u.s.c. Also for $x \in X, F(x)=\pi_{2}\left(\pi_{1}^{-1}(x) \cap F\right)$ is compact.

Remark. In particular, if $X$ is $T_{2}$ and $F$ is compact, then $F$ is u.s.c. and point compact.

LemMA 3.6. If the compact subsets of $X$ are closed and if $\alpha, \beta \subseteq X \times X$ are compact relations, then $D(\alpha \circ \beta)$ is compact.

Proof. By Lemma 3.5, $\beta^{-1}$ is u.s.c. and point compact. Now $D(\alpha)=\pi_{1}(\alpha)$ and $R(\beta)=\pi_{2}(\beta)$ are compact. Thus $D(\alpha) \cap R(\beta)$ is compact. Finally,

$$
D(\alpha \circ \beta)=\beta^{-1}(D(\alpha) \cap R(\beta))
$$

is compact by Lemma 3.2.

THEOREM 3.7. (Magill). If $X$ is $T_{2}$ and if $\alpha, \beta$ are compact relations in $X$, then $\alpha \circ \beta$ is a compact relation.

Proof. By Lemma 3.5 and a slight extension of Lemma 3.3, we get that $\alpha \circ \beta$ is u.s.c. and point compact. Further, by Lemma 3.6, $D(\alpha \circ \beta)$ is compact. Thus Lemma 3.4 applies. Hence, $\alpha \circ \beta$ is compact.

REMARK. The advantage of the new proof of Magill's result, and its display in the series of lemmas is that it leads us directly to the desired extension.

The next definition is from [8].

Definition. A topological space $X$ is a cc-space in case the compact subsets of $X$ are closed.

The proof of Theorem 3.8 is exactly the same as the proof of Theorem 3.7. 
THEOREM 3.8. Compactness is a composable property of the class of cc-spaces.

THEOREM 3.9. Let $X$ be a cc-space and let $\alpha \subseteq X \times X$. Then the following are equivalent:

(i) $\alpha$ is compact.

(ii) $\alpha$ is u.s.c. and point compact and $D(\alpha)$ is compact.

(iii) $\alpha, \alpha^{-1}$ preserve compact sets and $D(\alpha)$ is compact.

Proof. Lemma 3.4 is (ii) implies (i). Lemma 3.5 is (i) implies (ii) since $\pi_{1}(\alpha)=D(\alpha)$. So (i) and (ii) are equivalent. (This much generalizes the Compact Graph Theorem of Kolodner [3] to multifunctions.) Suppose (i) holds and $C$ is a compact subset of $X$. Then

$$
\alpha(C)=\pi_{2}\left(\pi_{1}^{-1}(C) \cap \alpha\right)
$$

is compact. Similarly, $\alpha^{-1}(C)=\pi_{1}\left(\pi_{2}^{-1}(C) \cap \alpha\right)$ is compact. So (iii) holds, and (i) implies (iii). Assume now that (iii) holds. Then $\alpha$ is point compact, $D(\alpha)$ is compact, and $R(\alpha)=\alpha(D(\alpha))$ is compact. Let $C$ be a closed subset of $X$. Then

$$
\alpha^{-1}(C)=\alpha^{-1}(C \cap R(\alpha))
$$

is compact and hence closed. Thus $\alpha$ is u.s.c. and (ii) holds.

ReMarKs. Lemma 3.3 and the equivalence of (i) and (ii) in Theorem 3.9 are exactly the reasons why compactness is a composable property of $c c$-spaces. The equivalence of (i) and (iii) in Theorem 3.9 sheds light on the connectedness setting and provides motivation for our choice of $S$ in section 2 . In the connectedness setting, the correspondent of Theorem 3.9 is completely false, and this single fact goes far in explaining why connectedness is not a composable property.

Now we need another definition and a result from Wattel [8].

Definition. A space $X$ is called a $c$-space in case a subset $A$ of $X$ is closed if and only if its intersection with each closed compact set is compact.

Lemma 3.10. (Wattel). A space is a c-space if and only if it is a cc-space and a $k$-space. So all locally compact $T_{2}$ spaces and all first countable $T_{2}$ spaces are c-spaces being $k$-spaces.

Proof. Suppose $X$ is a $c$-space and let $K$ be a compact subset of $X$. Then $K$ has a compact intersection with every closed compact set, and so $K$ is closed. Thus $X$ is a $c c$-space. If $A$ has a closed intersection with each closed compact set, then $A$ has a compact intersection with each closed compact set. Thus $A$ is closed, and so $X$ is a $k$-space. Conversely, if $X$ is a $c c$-space and a $k$-space and if $A$ has a compact intersection with each closed compact set, then $A$ has a closed 
intersection with each closed compact set. Thus $A$ is closed, and hence, $X$ is a $c$-space.

Now let $S[X]$ and $S[Y]$ be the compact relations in $X$ and $Y$, respectively.

Theorem 3.11. If $X$ and $Y$ are c-spaces, then $X$ is homeomorphic to $Y$ if and only if $S[X]$ is isomorphic to $S[Y]$.

Proof. By using Theorem 3.8 and Lemma 3.10, it is easy to see that $S[X]$ and $S[Y]$ are triform semigroups where $\mathscr{F}_{X}$ and $\mathscr{F}_{Y}$ are the compact subsets of $X$ and $Y$, respectively. Suppose that $S[X]$ and $S[Y]$ are isomorphic. By Theorem $A$ there is a bijection $h$ on $X$ onto $Y$ such that $h$ and $h^{-1}$ map compact sets onto compact sets. Finally, since $X$ and $Y$ are $c$-spaces, it is easy to show that $h$ and $h^{-1}$ are continuous. Thus $X$ and $Y$ are homeomorphic. Conversely, if $H: X \rightarrow Y$ is a homeomorphism, then $S[X]$ and $S[Y]$ are isomorphic by Theorem A because $\alpha \in S[X]$ implies $h \circ \alpha \circ h^{-1} \in S[Y]$ (Proposition 3.6 of [5]).

RemarK. If $X$ is a $c$-space, then the semigroups of relations defined by conditions (ii) and (iii) of Theorem 3.9 coincide with $S[X]$, and so they too suffice to characterize the space $X$.

\section{Continua}

We establish another composable property.

Definition. A multifunction $F: X \rightarrow Y$ is monotone if and only if $F^{-1}(y)$ is connected for each $y \in Y$. Equivalently, $F^{-1}: R(F) \rightarrow X$ is point connected.

Let $X$ be a space and let $S$ be the set of relations $\alpha$ in $X$ such that $\alpha$ is point connected, monotone and a continuum.

THEOREM 4.1. If $X$ is a cc-space, then $S$ is a semigroup of relations.

Proof. Let $\alpha, \beta \in S$. Then $\alpha, \beta$ are compact. By Theorem 3.9, $\beta$ is continuum valued and $\alpha$ is an u.s.c. continuum valued multifunction with $D(\alpha)$ a continuum. As mentioned ealier, $\alpha$ therefore preserves continua. Thus for $x \in X, \alpha \circ \beta(x)$ $=\alpha(\beta(x))$ is a continuum. Similarly, $(\alpha \circ \beta)^{-1}(x)$ is connected and so $\alpha \circ \beta$ is monotone. Now $D(\alpha \circ \beta)=\beta^{-1}(D(\alpha))$ is connected since $\beta^{-1}$ is u.s.c. (Theorem 3.9) and point connected. By Theorem 3.8, $\alpha \circ \beta$ is compact, and so $\alpha \circ \beta$ is an u.s.c. continuum valued multifunction with connected domain. Hence, $\alpha \circ \beta$ is connected and this completes the proof.

The following Theorem is an easy consequence of our previous work.

THEOREM 4.2. Let $X$ be a cc-space and let $\alpha \subseteq X \times X$. Then the following are equivalent:

(i) $\alpha$ is point connected, monotone and a continuum. 
(ii) $\alpha$ is u.s.c., continuum valued, and monotone and $D(\alpha)$ is a continuum.

(iii) $\alpha, \alpha^{-1}$ preserve both compact sets and connected sets and $D(\alpha)$ is a continuum.

Now let $S[X]$ and $S[Y]$ be those relations $\alpha$ in $X$ and $Y$ respectively, such that $\alpha$ is point connected, monotone and a continuum.

THEOREM 4.3. If $X$ and $Y$ are semi-locally connected, cc-spaces, then $X$ is homeomorphic to $Y$ if and only if $S[X]$ is isomorphic to $S[Y]$.

Proof. Since $X$ is a $c c$-space, Theorems 4.1 and 4.2 apply. Thus $S[X]$ and $S[Y]$ are triform semigroups where $\mathscr{F}_{X}$ and $\mathscr{F}_{X}$ are the subcontinua of $X$ and $Y$, respectively. Suppose that $S[X]$ and $S[Y]$ are isomorphic. By Theorem A there is a bijection $h$ on $X$ onto $Y$ such that $h$ and $h^{-1}$ map continua onto continua. But $X$ and $Y$ are semi-locally connected, and thus the proof of Theorem 9 of [6] applies showing that $h$ and $h^{-1}$ are continuous. Thus $X$ and $Y$ are homeomorphic. Conversely, if $h: X \rightarrow Y$ is a homeomorphism, then $S[X]$ and $S[Y]$ are isomorphic by Theorem A because $\alpha \in S[X]$ implies $h \circ \alpha \circ h^{-1} \in S[Y]$ (apply Theorem 4.2).

Remarks. Theorems 2.3, 3.11, and 4.3 all apply to trees. Finally, the relationship of $c c$ to $R_{0}$ is: $T_{2}$ implies $c c$ implies $T_{1}$ implies $R_{0}$. None of these implications reverse, in general.

\section{References}

[1] A. S. Davis, 'Indexed systems of neighborhoods for general topological spaces', Amer. Math. Monthly 68 (1961), 886-893.

[2] J. L. Kelley, General Topology (D. Van Nostrand, Princeton, New Jersey, 1955).

[3] I. I. Kolodner, 'The compact graph theorem', Amer. Math. Monthly 75 (1968), 167.

[4] K. D. Magill, Jr., 'Isomorphisms of triform semigroups', J. Austral. Math. Soc. 10 (1969), 185-193.

[5] K. D. Magill, Jr., 'Composable topological properties and semigroups of relations', J. Austral. Math. Soc. 11 (1970), 265-275.

[6] D. E. Sanderson, 'Relations among some basic properties of non-continuous functions', Duke Math. J. 35 (1968), 407-414.

[7] T. Tanaka, 'On the family of connected subsets and the topology of spaces', J. Math. Soc. Japan 7 (1955), 391-393.

[8] E. Wattel, The Compactness Operator in set theory and topology (Mathematisch Centrum, Amsterdam, 1968).

University of Wyoming

Laramie, Wyoming, USA 